\title{
An Evaluation of The Clinical And Etiological Profile of Acute Renal Failure in Obstetric Patients: An Observational Cross- Sectional Study
}

\author{
Dr. Pankaj Singh ${ }^{1}$,Dr. N. S. Sengar ${ }^{2}$,Dr. Sanjaya Sharma ${ }^{3}$,Dr.Medhavi Gautam ${ }^{4}$ \\ ${ }^{1}$ Assistant Professor, U.P.U.M.S. Saifai, Etawah, UP \\ ${ }^{2}$ Professor MLB Medical College Jhansi, UP \\ ${ }^{3}$ Professor, Department of Obstetric and Gynaecology, MLB Medical College, Jhansi , UP \\ ${ }^{4} \mathrm{MD}$ Medicine, Consultant
}

\begin{abstract}
Objective: To evaluate the clinical and etiological profile of acute renal failure (ARF) in obstetric patients in Bundelkhand region.

Methods: This was observational cross-sectional study. The women with previously healthy kidneys who developed renal complications during their pregnancy due to obstetrical causes were included in the study. A detailed clinical and etiological examination was done and recorded. The criteria for diagnosis of ARF was: urine output output $<400 \mathrm{ml} /$ day, history $<3$ month, bilateral normal sized kidney $(9-12 \mathrm{~cm})$ on ultrasonographic examination and abrupt rise of blood urea and serum creatinine.

Results: More than one third of patients were between 26-30 years (36\%). Multigravida (72\%) parity was observed in majority of patients. Fever (70\%) was the most common clinical symptom in ARF patients followed by uterine bleeding (60\%), hypotension (22\%), hypertension (18\%), Oliguric/anuric ARF (16\%). However, altered sensorium was seen in 14\% and jaundice in 12\%. Fluid overload and Diarrhoea each was in $10 \%$ ARF patients. The percentage of other clinical symptoms was less than 10\%. HELLP syndrome (32\%) was found to be the most common etiological factor in ARF patients followed by Falciparum malaria (30\%), sepsis (26\%), disseminated intravascular coagulation (18\%), pregnancy induced hypertension (16\%) and antepartum haemorrhage (14\%). The percentage of other etiological factors was less than 10\%.

Conclusion: Prevention is the best and least expensive solution to prevent ARF. Preventing sepsis, a good antenatal care and a better management of obstetrical complications are the crucial tools to implement to achieve the purpose.
\end{abstract}

Keywords: Obstetric patients, Acute renal failure, Etiology

\section{Introduction}

Pregnancy-related acute renal failure (ARF) is potentially life threatening, but largely preventable complication of pregnancy. A major decrease in its incidence in high- income countries has been attributed to the liberalization of abortion laws and improved obstetric care (Sulaniya et al, 2015). The incidence of pregnancy related acute kidney injury (PRAKI) has decreased in developed countries to only $1 \%$ to $2.8 \%$. It is a rare complication of pregnancy following the disappearance of septic abortion and a better prenatal care. However, PRAKI is still frequent in developing countries; the incidence is around 4.2-15\% (Mohamed et al, 2013).Depending on the severity and duration of the renal dysfunction, it can be accompanied by metabolic disturbances, such as metabolic acidosis and hyperkalaemia, changes in body fluid balance, and effects on many other organ systems. The care of pregnant women with renal failure is challenging because of consideration of wellbeing of mother and fetus. While Pregnancy Related-ARF (PR-ARF) has become rare occurrence in developed world, it continues to be associated with significant mortality and long term morbidity in developing world (Rajeshwari et al, 2015).

The present study was conducted to evaluate the clinical and etiological profile of acute renal failure in obstetric patients in north India.

\section{Material And Methods}

This was observational cross-sectional study conducted in a tertiary care hospital in north India. The study was approved by the Ethical Committee of the Institute. The written informed consent was taken from each participant before enrolling in the study. The women with previously healthy kidneys who developed renal complications during their pregnancy due to obstetrical causes were included in the study. Any patient with previous renal disease becoming pregnant was excluded from the study.A detailed clinical and etiological examination was done and recorded. The blood was collected from each patient and analysed for blood urea and 
serum creatinine level. Ultrasonographic was done in each patient. The criteria for diagnosis of ARF was: urine output output $<400 \mathrm{ml} /$ day, history $<3$ month, bilateral normal sized kidney $(9-12 \mathrm{~cm})$ on ultrasonographic examination and abrupt rise of blood urea and serum creatinine. The results are presented in percentages.

\section{Results}

More than one third of patients were between 26-30 years (36\%). Multigravida (72\%) parity was observed in majority of patients. More than one third (42\%) of ARF patients received antenatal care. The home delivery $(76 \%)$ was found in majority of ARF patients (Table-1).Fever (70\%) was the most common clinical symptom in ARF patients followed by uterine bleeding (60\%), hypotensin shock (22\%), hypertension (18\%), Oliguric/anuric ARF (16\%). However, altered sensorium was seen in $14 \%$ and jaundice in $12 \%$. Fluid overload and Diarrhoea each was in $10 \%$ ARF patients. The percentage of other clinical symptoms was less than $10 \%$ (Table-2).HELLP syndrome (32\%) was found to be the most common etiological factor in ARF patients followed by Falciparum malaria (30\%), sepsis (26\%), disseminated intravascular coagulation (18\%), pregnancy induced hypertension $(16 \%)$ and antepartum haemorrhage $(14 \%)$. The percentage of other etiological factors was less than $10 \%$ (Table-3).

\section{Discussion}

ARF may comprise up to $25 \%$ of the referrals to dialysis centers in developing countries and is associated with substantial maternal and fetal mortality (Hassan et al, 2009). In India, the rate of ARF related to the septic abortion had reduced from 33.3\% to $1.8 \%$ in last 20 years (Goplani et al, 2008). The high prevalence of anemia $(68.3 \%)$ among even married women of reproductive age in India could compromise their ability to tolerate hemorrhage /sepsis during abortion and. However, lack of prenatal care in India is more than a medical problem because associated socio-cultural, economic and geographic barriers often prevent women from receiving appropriate obstetric care (Arora et al, 2010). There have been many studies of the epidemiology of acute renal failure. However, in most cases, the definition of acute renal failure rests on arbitrary biochemical cut-off points. These biochemical dividing lines vary from study to study making comparisons difficult. In this study, more than one third of patients were between 26-30 years (36\%). Multigravida (72\%) parity was observed in majority of patients. In a study (Rajeshwari et al, 2015), the peak incidence of ARF was seen between 21 to 25 years and 31 to 35 years age group. Among 30 patients with ARF, $40 \%$ patients were primigravida and $30 \%$ patients were multigravida.

There is a significant increase in the incidence of hypertensive disorders of pregnancy contributing to high maternal and perinatal mortality (Rani et al, 2002). In the present study, $18 \%$ patients had history of hypertension. In Syed Munib et al (2008) study, ARF due to sepsis has fallen significantly in the last 30 years relative to the incidence secondary to obstetric complications (eg, abruption placenta, amniotic fluid embolism and post-partum Haemorrhage) in developed countries. In developing countries, the incidence of pregnancy related ARF is still high, mainly due to lack of education and antenatal care. Sepsis accounted for $26 \%$ in the present study followed by antepartum haemorrhage (14\%). In the present study, HELLP syndrome (32\%) was found to be the most common etiological factor in ARF patients followed by Falciparum malaria (30\%), disseminated intravascular coagulation (18\%) and pregnancy induced hypertension (16\%). Utas et al (2000) reported that etiology of the ARF related to the pregnancy $(n=70)$ were as follows; Eclampsia (50\%), HELLP Syndrome (15.7\%), post-partum Haemorrhage (14.3\%), septic abortion (11.4\%) and postpartum ARF (8.6\%). Kumar et al (2006), on the other hand, reported etiology of the ARF related to the pregnancy as follows $(n=74)$ : abortion (30\%), HELLP syndrome and pre eclampsia (14\%), pre eclampsia or eclampsia (12\%), post-partum Haemorrhage(15\%), IUD (12\%), abruption placenta (6\%), placenta previa (1\%).

In this study, oliguric/anuric ARF was seen in $16 \%$ and non-oliguric/anuric ARF was in $8 \%$. In a study (Rajeshwari et al, 2015), non oliguric ARF was seen in $66 \%$ of cases compared to $33 \%$ of oliguric cases. The difference might be due to difference in the socio-demographic profile between the two studies. One of the limitations of this study was the smaller sample size, the studies on larger sample size are recommended to have robust conclusions. Also, we did not included the cases without ARF, so the clear picture of prevalence/incidence could not measured.

\section{Conclusion}

Prevention is the best and least expensive solution to prevent ARF. Preventing sepsis, a good antenatal care and a better management of obstetrical complications are the crucial tools to implement to achieve the purpose.

Conflict of interest: None

Source of funding: None 


\section{References}

[1]. Sulaniya Chandrakanta, Sulaniya Pawan Kumar, Sharma Anju, Dixit Anand Mohan, Khuteta Rakesh Pratap, Naga Oby. An observational prospective study of clinical profile and obstetrical and neonatal outcome of Pregnancy Related Acute Renal Failure occurred in a tertiary care hospital of Rajasthan. IOSR Journal of Dental and Medical Sciences (IOSR-JDMS). 2015 ; 14 (3): 01-05.

[2]. Mohamed Arrayhani, Randa EiYoubi, and Tarik Sqalli. Prenancy -Related Acute Kidney Injury: Experience Of The Nephrology Unit At The University Hospital Of Fez, Morocco: Isrn Nephrology. 2013.

[3]. Rajeshwari S. N, Asha Swarup, Mahesh E. Obstetric Renal Failure. Journal of Evidence based Medicine and Healthcare. 2015; 2 (46): 8241-8251.

[4]. Hassan Irfan, Junejo Abdul Manan And Dawani Anohar Lal. Etiology And Outcome Of Acute Renal Failure In Pregnancy. Journal Of The College Of Physician And Surgeons Pakistan. 2009, 19: 714-717.

[5]. Goplani Kr, Shah Pr, Gera Dn, Gumber M, Dabhi M, Feroz A, Kanodia, Suresh S, Vanikar A V, Trivedi Hl. Pregnancy -Related Acute Renal Failure: A Single -Center Experience. Indian J Nephrol. 2008; 18: 17-21.

[6]. Arora Nalini, Mahajan Kirti, Java Narayan, Taraphder Abhijit : Pregnancy -Related Acute Renal Failure In Eastern India Int J Of Gynae \& Obstet. 2010; 111: 213 -216.

[7]. Rani PU, Narayen G, Anuradha. The journal of Obstetrics and Gynaecology of India. 2002; 52(1): 36-8.

[8]. Syed Munib, Sheraz Jamal Khan. Outcomes of pregnancy related acute renal failure, 2008 research gate.

[9]. Utas C, Yalcindag C,Taskpan H, Guven M, Oymak O, Yucesoy M. Nephrol Dial Transplant. 2000; 15; 152-5.

[10]. Kumar Kilari Sunil, Krishna Chinta Rama, Kumar Vishnubhotla Siva. Pregnancy Related Acute Renal Failure; J Obstet Gynaecol India. 2006; 56 (4): 308-310.

Table-1: General characteristics of the patients

\begin{tabular}{|l|c|c|}
\hline \multicolumn{1}{|c|}{ Characteristics } & No. $(\mathbf{n = 5 0})$ & \% \\
\hline Age in years & & 24.0 \\
\hline $20-25$ & 12 & 36.0 \\
\hline $26-30$ & 18 & 26.0 \\
\hline $31-35$ & 13 & 14.0 \\
\hline$>35$ & 7 & 72.0 \\
\hline Parity & & 28.0 \\
\hline Multigravida & 36 & \\
\hline Primigravida & 14 & 42.0 \\
\hline Antenatal care & 21 & 58.0 \\
\hline Received & 29 & \\
\hline Not received & & 76.0 \\
\hline Place of delivery & 38 & 24.0 \\
\hline Home & 12 & \\
\hline Hospital & & \\
\hline
\end{tabular}

Table-2: Clinical characteristics of acute renal failure (ARF) patients

\begin{tabular}{|l|c|c|}
\hline \multicolumn{1}{|c|}{ Clinical characteristics* } & No. $(\mathbf{n}=\mathbf{5 0})$ & \% \\
\hline Hypotension & 11 & 22.0 \\
\hline Hypertension & 9 & 18.0 \\
\hline Oliguric/anuric ARF & 8 & 16.0 \\
\hline Non-oliguic failure & 4 & 8.0 \\
\hline Uterine bleeding & 30 & 60.0 \\
\hline Fluid overload & 5 & 10.0 \\
\hline Jaundice & 6 & 12.0 \\
\hline Hyperpremesis gravidaram & 3 & 6.0 \\
\hline Fever & 35 & 70.0 \\
\hline Diarrhoea & 5 & 10.0 \\
\hline Altered sensorium & 7 & 14.0 \\
\hline
\end{tabular}

*Multiple response

Table-3: Etiological factors of acute renal failure (ARF) patients

\begin{tabular}{|l|c|c|}
\hline \multicolumn{1}{|c|}{ Etiological factors* } & No. $(\mathbf{n = 5 0})$ & \% \\
\hline Pregnancy induced hypertension & 8 & 16.0 \\
\hline Antepartum haemorrhage & 7 & 14.0 \\
\hline Postpartum haemorrhage & 3 & 6.0 \\
\hline Sepsis & 13 & 26.0 \\
\hline Disseminated intravascular coagulation & 9 & 18.0 \\
\hline Drugs & 4 & 8.0 \\
\hline HELLP syndrome & 16 & 32.0 \\
\hline Falciparum malaria & 15 & 30.0 \\
\hline Pyelonephritis/UTI & 3 & 6.0 \\
\hline Acute gastroenteritis & 4 & 8.0 \\
\hline Abortions & 3 & 6.0 \\
\hline
\end{tabular}

*Multiple response 